\title{
Glycogen Storage Disease Type Ia in Canines: A Model for Human Metabolic and Genetic Liver Disease
}

\author{
Andrew Specht, ${ }^{1}$ Laurie Fiske, ${ }^{2}$ Kirsten Erger, ${ }^{3}$ Travis Cossette, ${ }^{3}$ John Verstegen, ${ }^{4}$ \\ Martha Campbell-Thompson, ${ }^{5}$ Maggie B. Struck, ${ }^{6}$ Young Mok Lee, ${ }^{7}$ Janice Y. Chou, \\ Barry J. Byrne, ${ }^{3,8}$ Catherine E. Correia, ${ }^{2}$ Cathryn S. Mah, ${ }^{3,8}$ David A. Weinstein, ${ }^{2}$ \\ and Thomas J. Conlon ${ }^{3,9}$ \\ ${ }^{1}$ Department of Small Animal Clinical Sciences, University of Florida, Gainesville, FL 32610, USA \\ ${ }^{2}$ Glycogen Storage Disease Program, Division of Pediatric Endocrinology, Department of Pediatrics, University of Florida, \\ Gainesville, FL 32610, USA \\ ${ }^{3}$ Powell Gene Therapy Center, University of Florida, Gainesville, FL 32610, USA \\ ${ }^{4}$ Department of Large Animal Clinical Sciences, University of Florida, Gainesville, FL 32610, USA \\ ${ }^{5}$ Department of Pathology, Immunology and Laboratory Medicine, University of Florida, Gainesville, FL 32610, USA \\ ${ }^{6}$ Animal Care Services, University of Florida, Gainesville, FL 32610, USA \\ ${ }^{7}$ Section on Cellular Differentiation, PDEGEN, National Institute of Child Health and Human Development, \\ National Institute of Health, Bethesda, MD 20892, USA \\ ${ }^{8}$ Division of Cellular and Molecular Therapy, Department of Pediatrics, University of Florida, Gainesville, FL 32610, USA \\ ${ }^{9}$ Department of Pediatrics, University of Florida College of Medicine, P.O. Box 103610, Gainesville, FL 32610, USA
}

Correspondence should be addressed to Thomas J. Conlon, conlon@peds.ufl.edu

Received 6 October 2010; Accepted 24 November 2010

Academic Editor: Monica Fedele

Copyright () 2011 Andrew Specht et al. This is an open access article distributed under the Creative Commons Attribution License, which permits unrestricted use, distribution, and reproduction in any medium, provided the original work is properly cited.

A canine model of Glycogen storage disease type Ia (GSDIa) is described. Affected dogs are homozygous for a previously described M121I mutation resulting in a deficiency of glucose-6-phosphatase- $\alpha$. Metabolic, clinicopathologic, pathologic, and clinical manifestations of GSDIa observed in this model are described and compared to those observed in humans. The canine model shows more complete recapitulation of the clinical manifestations seen in humans including "lactic acidosis", larger size, and longer lifespan compared to other animal models. Use of this model in preclinical trials of gene therapy is described and briefly compared to the murine model. Although the canine model offers a number of advantages for evaluating potential therapies for GSDIa, there are also some significant challenges involved in its use. Despite these challenges, the canine model of GSDIa should continue to provide valuable information about the potential for generating curative therapies for GSDIa as well as other genetic hepatic diseases.

\section{Introduction}

Glycogen storage disease type Ia (GSDIa; von Gierke disease; MIM 232200) is an inherited metabolic disorder resulting from a deficiency in the enzyme glucose 6-phosphatase$\alpha$ (G6Pase; EC 3.1.3.9). Without G6Pase activity, all endogenous glucose production is impaired as this critical enzyme catalyzes the final step of both gluconeogenesis and glycogenolysis. Consequently, circulating blood glucose levels cannot be increased in response to positive glucoregulatory stimuli leading to a condition characterized by fasting hypoglycemia, as well as accumulation of glycogen and fat, particularly within liver and kidney tissues [1-3]. Shunting of glucose-6-phosphate (G6P) into alternative metabolic pathways results in lactic acidosis, hypertriglyceridemia, and hyperuricemia [1-3].

Current therapy is directed at preventing hypoglycemia through sustained provision of glucose via continuous or frequent feedings or consumption of uncooked starches [4-8]. These types of palliative dietary therapy have had a profound impact on morbidity and mortality, allowing most affected individuals to have near normal growth, 
pubertal development, and subsequent survival to adulthood. However, the underlying pathology remains untreated and the therapy can have other metabolic consequences such as hyperinsulinemia and excessive caloric intake from continuous glucose delivery [9]. In addition, long-term complications remain a problem in individuals with GSDIa [10-13]. Therefore, a search for additional or alternative therapeutic approaches geared to help improve quality of life and long-term outcomes for patients with GSDIa continues. Gene therapy or stem cell therapy is expected, by correcting the underlying problem of G6Pase deficiency, to prevent the complications of GSDIa and the undesired consequences of current therapies, thereby improving the prognosis for such patients. Evaluation of the potential safety and efficacy of such therapies requires testing in appropriate animal models.

A murine model of GSDIa has been generated which manifests most of the clinical signs and much of the pathology associated with the human condition $[14,15]$. However, this model has a few drawbacks including small size of the animals and a short lifespan. In addition, there are also some key differences between the disease manifestation in humans and the GSDIa knockout mouse. First, mice with GSDIa have not been shown to develop lactic acidosis until they are at least 6 weeks of age and the difference in blood lactate concentrations between wild type and affected mice reported after this age is mild compared to that observed in human patients [14-16]. Secondly, in contrast to humans, adenoma formation at equivalently young ages is uncommon. Thus, while this model has proven very useful in furthering our understanding of GSDIa, an animal model with physiology and long-term consequences closer to what is observed in humans is advantageous before the experimental techniques can safely be attempted in humans.

A naturally occurring canine model has been described with clinical manifestations that recapitulate most of the features of GSDIa seen in humans including profound lactic acidosis [17-19]. The purpose of this paper is to review important aspects of human GSDIa and to provide a detailed comparison to the canine model. In addition, an emphasis will be placed on how the canine model is currently being used in preclinical trials for gene therapy mediated correction of GSDIa and how such research can contribute to the development of new potential therapies and cures for this and possibly also other genetic and metabolic disease conditions.

\section{Genetic Basis for GSDIa and Diagnosis}

The G6Pase complex is located in the inner membrane of the endoplasmic reticulum. In order for glucose-6-phosphate to be hydrolyzed to glucose, G6P must be transported through a bidirectional translocase to the catalytic site. GSDIa occurs when the G6Pase is not produced or when mutations result in a nonfunctional enzyme. The gene encoding the catalytic unit was identified in band q21 of chromosome 17 in humans [20]. Over 85 different mutations causing GSDIa have been described in humans, for which the typical metabolic disturbances are fairly consistent [20-24].
The gene for the catalytic unit of the G6Pase complex in canines has been identified and demonstrates significant homology when compared to humans, mice, and rats [18]. The DNA sequence contains $2346 \mathrm{bp}$ with a $5^{\prime}$ untranslated region of $87 \mathrm{bp}$, an open reading frame of $1071 \mathrm{bp}$, and a 3' untranslated region of $1185 \mathrm{bp}[18,19]$. The open reading frame encodes a 357 amino acid sequence with $91.3 \%$ homology to the 357 amino acid sequence encoded by the human gene [18]. The specific mutation in affected dogs is a guanine to cytosine transversion at nucleotide position 450 (G450C) [18]. This results in substitution of a methionine by isoleucine at codon 121 (M121I) [18]. Of note, the location of the canine mutation is very close to a known human mutation in exon 4 of the gene and subsequent transfection studies have proven that the mutant G6Pase has 15 times less enzyme activity than normal [18-24].

Current breeding colonies of the canine model originated from Maltese dogs expressing the carrier (heterozygous) state for this naturally occurring mutation [19]. These Maltese dogs were eventually cross-bred to normal (wild-type) Beagles to help increase the average size of the individual dogs and consequently the average litter size. As in humans, only dogs homozygous for the M121I mutation demonstrate the overt clinical manifestations of GSDIa $[18,19]$.

Genetic testing can be easily performed on blood or saliva from dogs using PCR and direct sequencing of the exon as previously described (Figure 1(a)). In addition, digestion with the restriction enzyme NcoI can be used to distinguish affected, carrier, and wild-type dogs. Since the restriction enzyme cannot cut the mutated sequence, affected $(-/-)$ dogs have a larger fragment compared to wild-type dogs (Figure 1(b)).

\section{Metabolic Disturbances in GSDIa}

Glucose is the primary energy source for most mammalian cells, and its metabolism is tightly regulated to guarantee that a sufficient supply is available to glucose-dependent organs, particularly the brain. Glucose can be made available from two sources: absorption of dietary glucose from the intestine and release of glucose from organs such as the liver or kidney. Early in fasting, the majority of endogenous glucose is generated by glycogenolysis where glycogen in the liver is converted to G6P under the regulation of debranching enzyme, hepatic glycogen phosphorylase, and phosphorylase kinase. With more prolonged fasting, endogenous glucose is generated by gluconeogenesis from certain substrates such as amino acids, lactate, and glycerol. Both processes generate G6P which must then be dephosphorylated in order to transport glucose out of the cell (Figure 2(a)). The enzyme responsible for this is G6Pase alpha. Alterations in quantity, location, or activity of G6Pase such as those seen in type I glycogen storage diseases effectively result in a lack of all endogenous glucose production and severe hypoglycemia develops during periods of fasting (Figure 2(b)).

The high concentrations of G6P generated in GSDIa are ultimately shunted into alternative pathways causing the classic triad of hyperlipidemia, hyperuricemia, 


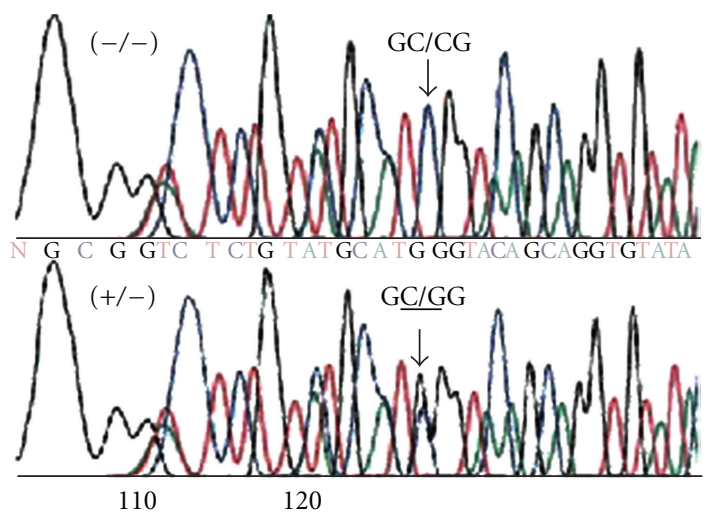

(a)

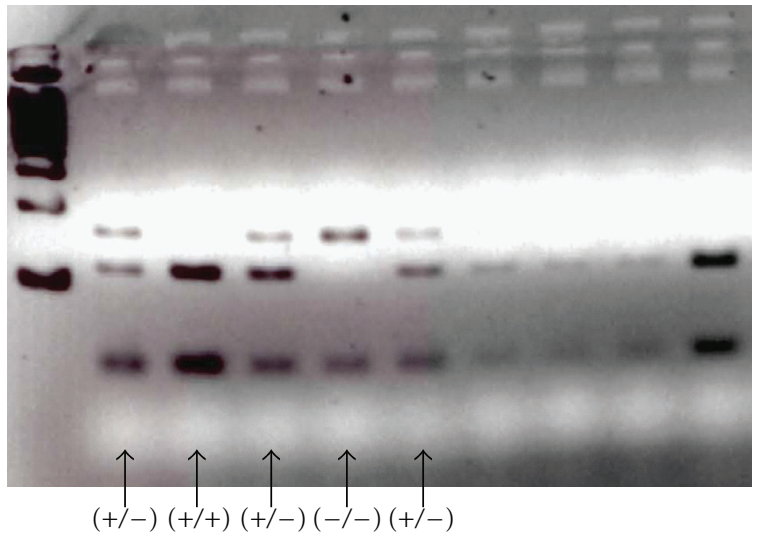

(b)

FIGURE 1: PCR sequencing and restriction digest for the canine mutation. (a) Sequencing chromatogram of the mutation region showing the G450C transversion on both alleles of an affected $\operatorname{dog}(-/-)$ and on one allele in a heterozygote (+/ - ). (b) Ncol digest after amplification of the target region resulting in cutting of the wild-type alleles $(+/+)$, cutting of one allele in the heterozygote $(+/-)$, and larger undigested bands from both alleles in the homozygous dog $(-/-)$.

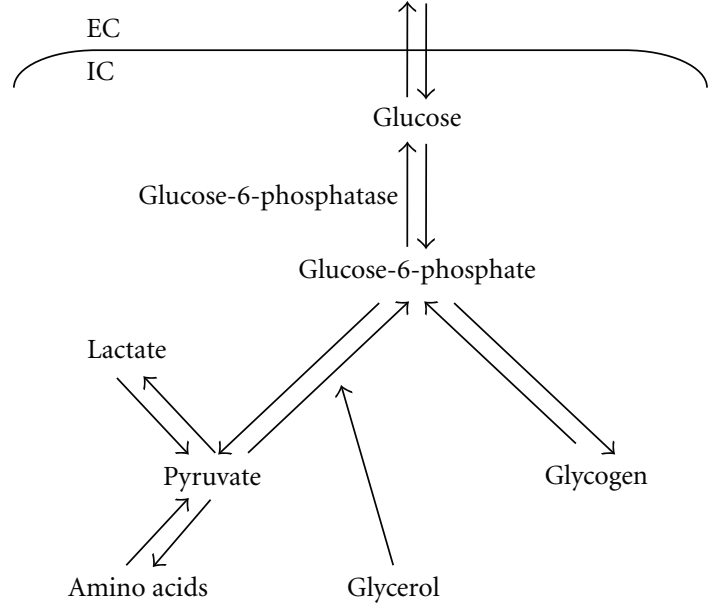

(a)

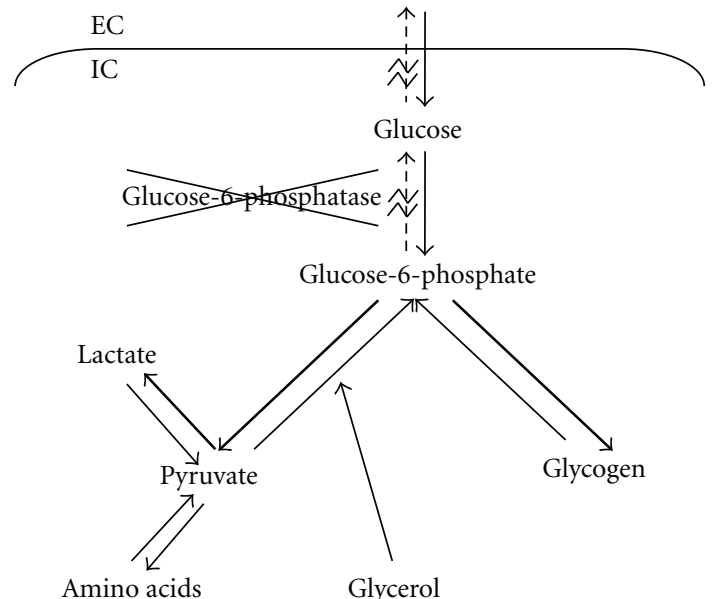

(b)

FIgURE 2: Outline of endogenous glucose production pathways. EC = extracellular, IC = intracellular. (a) Normal pathway and function of G6Pase. (b) Alterations seen with deficiency of G6Pase (GSDIa) include lack of dephosphorylation of G6P and shunting of excess G6P to produce glycogen and lactate.

and hyperlactatemia. Elevated lactate concentrations result from shunting down the glycolytic pathway resulting in a metabolic acidosis. Hyperlipidemia is a result of increased synthesis of triglycerides from shunting to acetyl CoA, inhibition of carnitine palmitoyltransferase I by malonyl CoA, and decreased lipid serum clearance. Shunting of G6P into the pentose phosphate pathway and increased degradation of adenine nucleotides result in increased uric acid production. Competitive inhibition of uric acid excretion by lactate results in decreased renal clearance.

As hypoglycemia develops in GSDIa, persistent alterations in several glucoregulatory hormones occur, most notably increased glucagon levels and decreased insulin levels. There may be alterations in epinephrine or cortisol as well, and this stimulation continues to worsen the metabolic alterations. If dietary glucose is available on a relatively constant basis as observed in many treated GSDIa patients, glycogen degradation and gluconeogenesis decrease, and metabolic abnormalities are expected to diminish.

\section{Pathology, ClinicoPathologic Findings, and Clinical Manifestations of GSDIa}

4.1. Without Treatment. Type Ia GSD is a disease that affects approximately 1 in 100,000 individuals. The disease was first described by von Gierke in 1929, but it was almost universally fatal marked by severe hypoglycemia, growth retardation, and life threatening acidosis prior to the introduction of continuous glucose therapy in 1971 [25-27]. While continuous feeds through a nasogastric or gastrostomy tube allowed 
children with this disease to survive, any interruption of the feeds resulted in rapid onset of hypoglycemia. Seizures and deaths related to hypoglycemia remained common until uncooked cornstarch (UCCS) therapy was introduced in $1982[4,5,28,29]$. As a result of these dietary interventions, the prognosis for humans with GSDIa has improved dramatically, but complications including metabolic acidosis due to a persistent elevation in blood lactate levels, hepatic adenoma rarely leading to hepatocellular carcinoma, chronic progressive kidney disease, gout, osteoporosis, and pulmonary hypertension remain common in adolescent and adult patients [7, 9-13].

There are little data about completely untreated (e.g., no dietary supplementation) dogs with GSDIa. The canine disease was originally described in two untreated puppies that died or were euthanized at about 47 days of age (about the time they would have been expected to begin weaning), and the authors are not aware of any reports of any untreated dogs having lived past this age [17]. The same litter had included a mummified fetus, another littermate that died at about 35 days of age, and one clinically healthy puppy that weighed almost 2.5 times the affected puppies at the same age. There are no published reports of antemortem diagnostic testing performed in these two dogs, but the medical history included notes of markedly reduced growth rate and poor body condition, mental depression, abdominal distension, hepatomegaly, and nephromegaly.

Gross pathologic findings from necropsy of both puppies included emaciation, enlarged, pale and friable livers, enlarged and pale kidneys with white lines present along the renal crest and at the corticomedullary junction, mild hydrocephalus, and enlarged, rounded spleens [17]. Histologic lesions were similar in both puppies. There was severe, diffuse vacuolar change in hepatocytes resulting in distortion of normal hepatic architecture. In the kidneys, there was evidence of interstitial fibrosis, and moderate vacuolar change in tubular epithelial cells. Cytoplasm from vacuolated hepatocytes and renal epithelium demonstrated positive periodic acid-Schiff (PAS) staining (consistent with glycogen storage) but were negative for Sudan staining (indicating that the vacuoles did not contain fat). Mineralization was also noted in the kidney, especially in proximity to the renal pelvis and along the corticomedullary junction, and also in the lung within the alveolar septa. Other changes included extramedullary hematopoiesis in the spleen and small follicles in the thyroid gland that were devoid of colloid.

Glycogen, G6Pase, and a few other metabolic enzyme levels in the liver and kidney tissue of these two affected puppies were compared to control puppies that died at similar ages from other diseases. Glycogen levels were significantly increased in the affected puppies whereas G6Pase levels were severely reduced. There was no significant difference noted in the other tested metabolic enzymes.

4.2. With Dietary Intervention. Although current dietary treatment protocols have dramatically improved survival and quality of life for humans with GSDIa, the underlying G6Pase deficiency remains. People with glycogen storage disease remain susceptible to rapid development of hypoglycemia should therapy be interrupted. Most adults continue to require 5-6 doses of UCCS per day to maintain good metabolic control, although review of actual patient intakes have demonstrated that some adult patients receive less frequent doses of UCCS [7, 11, 30, 31]. In addition, complications of the uncorrected enzyme deficiency and metabolic changes associated with dietary interventions are a source of significant morbidity during and after adolescence [10-13].

In the canine population, all untreated dogs with GSDIa die and all long-term survivors are part of gene therapy research trials [17-19, 32-34]. Attempts at maintaining dogs using nutritional therapies similar to those used in affected humans have proven unsuccessful, and long-term nutritional therapy has not been reported to date in the literature. In our experience, nutritional therapy was able to keep a dog alive to 5-1/2 months, but chronic acidosis, respiratory distress, and intermittent hypoglycemia remained problematic even with feeds administered every 30 minutes [34]. Hyperlipidemia and hepatomegaly worsened over time, and ultimately this dog developed severe hepatic lipidosis and pancreatitis [34].

Notably, unlike the aforementioned murine model, dogs with GSDIa continue to have fasting hypoglycemia after weaning $[30,32]$. Although the overall success of nutritional intervention seems to be lower for the canine model than for human patients at the current time, puppies with GSDIa appear to have similar pathologic changes and many of the same clinical manifestations of disease that are seen in human GSDIa patients [17-19, 25-27, 29, 32-34].

Fetal mortality and whelping complications are extremely common in canine GSDIa. Stillbirth, fetal resorption, and mummification are all frequently encountered problems in litters from heterozygote parents, and up to $50 \%$ of GSDIa puppies have been reported to be stillborn or die in the perinatal period [19]. Affected puppies are hypoglycemic at birth, and can struggle with nursing [32]. Without intervention, almost all affected puppies die within an hour of birth. With initiation of feeds, hepatomegaly rapidly develops. In order to prevent severe hypoglycemia and seizures, puppies must be manually feed every 30 minutes, but they continue to struggle with poor growth rates, lower activity levels, decreased mental alertness, and delayed development of neurologic reflexes $[17,19]$. Severe hypoglycemia to less than $35 \mathrm{mg} / \mathrm{dL}$ develops within 60 minutes of a feed if therapy is interrupted, and biochemical abnormalities in affected puppies include persistently elevated levels of lactate, uric acid, cholesterol, and triglycerides [19, 34].

In puppies that have died (or were euthanized) despite receiving nutritional support for their GSDIa, pathologic lesions have been identified that were similar, but not identical, to those of untreated puppies $[19,34]$. All puppies had gross hepatomegaly ranging from mild to severe, but renomegaly was not noted $[19,34]$. Histopathology revealed marked hepatocellular vacuolar changes with distortion of normal architecture and positive PAS and oil-red-O staining, demonstrating the presence of both glycogen and fat $[19,34]$. Vacuolar changes suggestive of glycogen storage were also seen in renal tubular epithelium, and glomerular changes 
were also observed in the slightly older ( $>30$ days) dogs [19].

One potential reason for the limited success of dietary interventions in puppies with GSDIa is the lack of information about the specific nutritional needs of these dogs. Affected neonates have been managed with a variety of dietary protocols that included allowing for nursing but provided additional carbohydrate supplementation in the form of milk replacer formula, glucose polymers, or dextrose given orally, or by parenteral administration of dextrose containing fluids [19, 32, 34]. Postweaning untreated, affected puppies and adults have been managed with frequent feedings (q2-4 hours) of commercially available puppy foods with additional carbohydrate support in the form of milk replacer formula, glucose polymers, UCCS, or dextrose given orally at frequent intervals ( $\mathrm{q} 30 \mathrm{~min}$ to $\mathrm{q} 4$ hours) $[19,32,34]$. Protocols are still being developed to help identify the best delivery methods (i.e., oral feeding, gavage feeding, or various types of feeding tubes) and optimal nutritional profiles to provide adequate glucose without sacrificing other important nutrients. It is hoped that with more experience and better treatment protocols affected puppies from future litters will have less developmental problems and improved survival to adulthood.

Because so few affected dogs have survived to adulthood, it is difficult to determine if many of the long-term complications seen in human patients will be recapitulated by this model. Hopefully, as the dog colonies grow and better nutritional protocols are developed, a group of affected adult dogs can be followed to document and characterize the complications that occur in this model.

4.3. Heterozygotes. Although only dogs that are homozygous for the M121I mutation exhibit the overt clinical manifestations of GSDIa, there are some data from our colony to suggest that dogs that are heterozygous for the mutation have subtle manifestations of the disease. Heterozygote dogs have $\sim 50 \%$ of the G6Pase activity of wild-type individuals [34]. They are able to tolerate a 12-hour fast with no apparent clinical signs and without hypoglycemia [34]. However, after a 12-hour fast these dogs do have slightly elevated blood lactate levels compared to wild-type dogs and laboratory reference standards [34]. Liver biopsies reveal that these dogs have mild vacuolar changes with positive PAS staining and MRS data from one dog showed about 2.9 times greater levels of glycogen in the liver than was found in a wild-type dog [34]. These finding suggest that although heterozygote dogs exhibit an overall normal outward phenotype, $50 \%$ of wildtype G6Pase activity does not provide a completely normal biochemical, physiological, or histological phenotype.

The human carrier population for various mutations in the G6Pase gene has not been reported to exhibit any overt pathology or complications of GSDIa. Whether the specific abnormalities detected in our heterozygote dogs also occur in the human population remains unknown at this time. There are some parents of affected children who have provided anecdotal accounts of symptoms such as shaking and weakness with fasting. These could be incidental or could be attributed to similar subtle clinical abnormalities related to comparatively lower levels of G6Pase.

\section{Use of the Canine GSDIa Model for Preclinical Trials}

Currently, the canine model of GSDIa is being studied primarily as part of early stage preclinical trials of recombinant adeno-associated virus (AAV) vector-based therapies. These types of therapy offer great promise for the treatment of GSDIa since they target the underlying problem rather than just palliating some of the clinical effects of G6Pase deficiency.

With the ability to generate large numbers of affected animals quickly, the murine model of GSDIa has provided a useful platform to prove the concept of gene therapy for correction of GSDIa and to compare the efficacy of various types of vectors $[33,35,36]$. Gene therapy has mediated prolonged survival, sustained correction of glucose homeostasis and normalization of triglycerides, cholesterol, and uric acid in mice using a variety of different vectors, although substantial variation in efficacy and specific effects of the different vectors has been identified [33, 35-38]. The lack of ability to address all of the clinical manifestations (e.g., lactic acidosis) of GSDIa in this model and the lack of ability to address long-term safety and efficacy due to the short overall lifespan of the mice demonstrate the need for additional studies in a different model.

The first reported results of gene therapy in the canine model utilized an AAV vector administered between 34 days after birth [32]. Puppies that received the vector demonstrated increased G6Pase activity in the liver and decreased glycogen compared to untreated, affected dogs. Some dogs also had improvement in blood glucose, triglyceride, cholesterol, and lactate levels after a three-hour fast compared to untreated, affected dogs at a least one time point after vector administration. When compared to carriers or wild-type dogs; however, these treated dogs demonstrated only partial improvements in biomarker levels and all succumbed to a GSDIa-related medical crisis between 20-86 days of age.

Later reports indicated that gene therapy utilizing an AAV2/8 vector significantly improved survival in three dogs compared to untreated controls and the dogs from the previous report by Beaty et al. [32, 33]. All three dogs survived passing 11 months of age. In addition to improved survival, treated dogs were able to maintain normal blood glucose levels during a fasting period of at least 2 hours (longer times are not reported) starting after 1-6 month (s) of age. While treated dogs still required frequent feeding (q6-10 hr) compared to normal dogs, they did not require additional carbohydrate supplementation. Glycogen and fat storage in the liver was reduced, and G6Pase activity was increased compared to values from untreated dogs and were comparable to levels reported in carrier dogs which showed no overt clinical signs of GSDIa. While lactate levels after a 2-hour fast were significantly improved in the treated dogs compared to untreated controls, they remained well above normal laboratory standards for dogs $(>2.1 \mathrm{mmol} / \mathrm{L})$.

Several dogs from a colony at the University of Florida have also been treated with gene therapy utilizing AAV vectors. The first dog was treated with an AAV2/8 vector on 
postnatal day 1 [34]. Two dogs from each of two subsequent litters have received gene therapy with AAV vectors at postnatal days 1 or 2 . Four of five treated dogs demonstrated an ability to maintain normal glucose and lactate levels during a two-hour fast at one month after treatment. The other dog did not receive the entire IV dose of vector due to subcutaneous extravasation and remained unable to tolerate a 2-hour fast.

While the dogs clinically improved after the initial dose of gene therapy, this level of correction was not sustained in any of these dogs. By two months of age, fasting glucose concentrations fell to levels similar to those of an untreated puppy and elevated lactate concentrations were consistently seen with fasting. In addition, growth curves for these dogs remained more similar to untreated controls than to wildtype puppies during the first few months of life. Similar results are reported in mice, in which intravenous delivery of AAV vector to deliver the gene of interest to neonates did not result in sustained correction.

Due to lack of sustained correction, the first dog was treated again at 20 weeks of age with a second dose of gene therapy delivered by an AAV2/1 vector injected into the portal vein [34]. This resulted in a sustained partial correction of the G6Pase deficiency and a dramatic improvement in the metabolic status of the dog. One month after treatment with the AAV2/1 vector, dextrose supplementation was discontinued and this dog continues to do well clinically three years later receiving a mixture of canned and dry preparations of a commercially available dog food supplemented with UCCS offered every 4-6 hours. Serial fasting studies show the best response at two months after treatment, when fasting blood glucose and lactate levels were normal for greater than 5 hours and still close to normal at 9 hours into the fast. The response was somewhat diminished at 15 months after injection, but in contrast to dogs described in prior reports, this dog has consistently maintained normal blood glucose and lactate levels throughout fasting periods of 2-4 hours. Liver biopsies collected at 30 months had greater G6Pas activity and much less glycogen and fat accumulation than biopsies from an untreated control; however, they were still abnormal when compared to wild-type controls.

The second and third dogs were doing well clinically until developing signs of acute respiratory distress at 74 days of age. Dyspnea and hypoxemia developed rapidly, and the dogs died from this complication despite aggressive critical care. Necropsy and infectious disease testing revealed severe viral pneumonia caused by Canine adenovirus-2 (which is distinct from, and unrelated to, the adeno-associated virus used as a vector). The death of these dogs was not deemed to be related to the gene therapy.

Two other dogs from our colony have recently been treated and have shown results similar to the first dog. They received their second doses of AAV vector at approximately 10 weeks of age. Both dogs are clinically healthy with no glucose supplementation at this time. However, these dogs are currently only five months old, so it is too early to determine if there will be a sustained partial correction of their condition.
Overall, the collective work of the two centers currently using the canine model of GSDIa in preclinical trials of AAV vector-mediated gene therapy have demonstrated significant improvements in biomarker levels and histopathologic abnormalities, and dramatically improved survival times for dogs treated with gene therapy compared to controls [33, 34].

\section{Challenges Involved in Use of the Canine GSDIa Model}

Despite a number of advantages that the canine model of GSDIa has over the murine model in studies evaluating potential therapies for human GSDIa, there are also some significant challenges involved in the use of the canine model for preclinical studies.

One challenge is generating a high number of affected dogs enough to make valid comparisons between different types of therapies. Affected dogs have not been reported to survive to adulthood without treatment, and so all breeding animals to date have been carriers (heterozygotes). Due to the reproductive biology of the dog, regulatory considerations, fertility issues related to GSDIa, and the expectation that only about $25 \%$ of offspring will be homozygotes; each breeding female in the colony produces an average of one affected puppy per year. Perinatal death is also an issue if dogs are allowed to whelp, and up to 50\% mortality has been reported in GSD dogs born naturally [19]. All litters in the colony at the University of Florida have been delivered by Cesarean section allowing $100 \%$ survival of affected puppies since dogs are immediately resuscitated with glucose upon birth, but this increases the cost and labor associated with having puppies with this disease.

The length of time needed to generate a reasonably sized dog colony presents some additional challenges. Each affected or carrier dog that is part of the colony needs housing, food, and intensive, around-the-clock care. Affected dogs can also experience medical crises with little warning. One refused feeding or an episode of vomiting or diarrhea can lead to severe hypoglycemia and lactic acidosis. Severe hypoglycemia can result in lethargy, weakness, loss of consciousness, or seizures. Lactic acidosis can cause or exacerbate anorexia, nausea, and vomiting which further complicates oral glucose administration. Most medical crises in GSDIa dogs require immediate additional oral glucose supplementation or parenteral administration of dextrose. Due to the fragility of GSDIa dogs, there is a need for oncall veterinary support at all times. The coordinated involvement of researchers, caretakers, and veterinary specialists is required to optimize the growth and health of the colony and the GSDIa gene therapy research. These factors also contribute to making the canine GSDIa model financially expensive to use.

Another major challenge in current GSDIa gene therapy research using the canine model is the lack of defined protocols proven to be effective for nutritional support of affected dogs. Currently, nutritional supplementation has not been reported to provide the same survival benefit to 
dogs with GSDIa that it has for people [4-11, 13]. The lack of comparable benefits so far may be due in part to a relative lack of experience treating dogs with GSDIa and the natural challenges that exist when translating human GSDIa nutrition protocols to a canine model. For example, growing dogs typically get a much higher percentage of energy from fats and proteins than do humans [39]. Since protein and fats cannot be used as a precursor of gluconeogenesis in dogs with GSDIa, affected dogs must necessarily receive a carbohydrate-heavy diet, which includes excessive amounts of carbohydrate compared with what normal dogs require. It is, therefore, difficult to provide a complete and balanced diet without overfeeding.

\section{Future Directions}

One target of future research will be improvement of nutritional support protocols to better meet the specific needs of dogs with GSDIa. Use of well defined nutritional support protocols that mimic protocols used in human GSDIa patients, but which are tailored to the specific needs of the canine model, will provide a comparison group for future preclinical trials of curative therapies, that is, a closer analogue to the human situation. Better nutritional management will also likely allow for a reduction in the cost of care for these dogs, improve survival times, and potentially allow affected dogs to participate in the breeding program which would significantly increase the number of affected and carrier puppies per litter.

Further work to identify an optimal protocol for gene therapy in GSDIa dogs including the best vector serotype, gene promoter, and timing and route of vector delivery hold great promise for developing a treatment that can eventually be translated for use in human GSDIa patients. Additional preclinical trials involving stem cell therapy or administration of novel longer-acting starches in this model may also yield valuable information about other potential therapeutic options for GSDIa.

Lastly, although GSDIa is a rare condition, preclinical trials of gene therapy and stem cell therapy to treat GSDIa in dogs will also serve as a useful model for testing the potential of these treatments for other hepatic genetic and metabolic diseases. There are over 30 different liver related disorders due to single-point mutations [40]. Many of these disorders require treatment with therapeutic agents that are expensive or difficult to obtain, and the efficacy of new treatment modalities can only be assessed through long-term studies and liver biopsies. In contrast, blood glucose and lactate serve as useful and inexpensive biomarkers for testing the success of therapy for GSDIa in canines and pretreated or control dogs can be managed with carbohydrate support. Thus, testing the feasibility and efficacy of new therapies in this model may then allow for translation to curative treatments for other genetic liver diseases.

\section{Conclusion}

The canine model of GSDIa has a similar genetic and underlying metabolic basis compared to GSDIa in humans.
Clinicopathologic, pathologic, and clinical manifestations of GSDIa in this model are also very similar to what is observed in humans, including lactic acidosis which is not a consistent feature of the murine model. It remains to be seen whether long-term complications of GSDIa seen in humans treated with the current standard of care for nutritional supplementation are also features of the canine disease. More complete recapitulation of the clinical manifestations of GSDIa in humans, larger size, and longer life span of dogs with GSDIa relative to mice are all advantages for use in preclinical trials of new treatments. However, there are some significant challenges as well such as the time, labor, and cost involved in generating useful numbers of affected dogs. Despite these challenges, preclinical trials have already demonstrated the potential for gene therapy to establish prolonged partial correction of the underlying G6Pase deficiency in canine GSDIa. With larger colonies, improved nutritional protocols, and additional preclinical trials, the canine GSDIa model should provide valuable information relating to optimization of gene therapy protocols and evaluation of other therapies such as stem cell therapy or novel starches. Eventually, research involving dogs with GSDIa may help to identify curative therapies which can then be translated to human patients with GSDIa or other hepatic genetic or metabolic diseases.

\section{Acknowledgments}

The authors gratefully acknowledge the UF GSD Puppy Care Team and the University of Florida Animal Care Services and College of Veterinary Medicine Veterinary Staff for their assistance in animal care; they also acknowledge the University of Florida Molecular Pathology Core; and the University of Florida Powell Gene Therapy Center Toxicology Core. This work was supported by Grants from the Children's Fund for Glycogen Storage Disease Research, the Children's Miracle Network, and the National Institutes of Health (nos. NHLBI P01 HL59412-06, NIDDK P01 DK5832703). Additional philanthropic support was provided from the Scott Miller Glycogen Storage Disease Program Fund, Matthew Ehrman GSD Research Fund, the Type Ib Glycogen Storage Disease Fund, the Jonah Pournazarian Type Ib GSD Fund, Green Family Fund for GSD Research, HLH Fund, and the Canadian Fund for the Cure of GSD. B. J. Byrne the Johns Hopkins University, and the University of Florida could be entitled to patent royalties for inventions described in this paper. D. A. Weinstein and T. Cossette share senior authorship for this work.

\section{References}

[1] Y. T. Chen and A. Burchell, "Glycogen storage diseases," in The Metabolic and Molecular Basis of Inherited Disease, C. Schriver and A. Beaudet, Eds., pp. 905-934, McGraw-Hill, New York, NY, USA, 1995.

[2] J. Y. Chou, D. Matern, B. C. Mansfield, and Y. T. Chen, "Type I glycogen storage diseases: disorders of the glucose-6phosphatase complex," Current Molecular Medicine, vol. 2, no. 2, pp. 121-143, 2002. 
[3] J. I. Wolfsdorf and D. A. Weinstein, "Glycogen storage diseases," Reviews in Endocrine and Metabolic Disorders, vol. 4, no. 1, pp. 95-102, 2003.

[4] G. P. A. Smit, R. Berger, and R. Potasnick, "The dietary treatment of children with type I glycogen storage disease with slow release carbohydrate," Pediatric Research, vol. 18, no. 9, pp. 879-881, 1984.

[5] G. P. A. Smit, M. T. Ververs, B. Belderok, M. Van Rijn, R. Berger, and J. Fernandes, "Complex carbohydrates in the dietary management of patients with glycogenosis caused by glucose-6-phosphate deficiency," American Journal of Clinical Nutrition, vol. 48, no. 1, pp. 95-97, 1988.

[6] J. Fernandes, J. V. Leonard, S. W. Moses et al., "Glycogen storage disease: recommendations for treatment," European Journal of Pediatrics, vol. 147, no. 3, pp. 226-228, 1988.

[7] D. A. Weinstein and J. I. Wolfsdorf, "Effect of continuous glucose therapy with uncooked cornstarch on the long-term clinical course of type la glycogen storage disease," European Journal of Pediatrics, vol. 161, no. 1, pp. S35-S39, 2002.

[8] K. Bhattacharya, R. C. Orton, X. Qi et al., "A novel starch for the treatment of glycogen storage diseases," Journal of Inherited Metabolic Disease, vol. 30, no. 3, pp. 350-357, 2007.

[9] H. R. Mundy, P. C. Hindmarsh, D. R. Matthews, J. V. Leonard, and P. J. Lee, "The regulation of growth in glycogen storage disease type 1," Clinical Endocrinology, vol. 58, no. 3, pp. 332339, 2003.

[10] G. P. A. Smit, J. Fernandes, J. V. Leonard et al., “The long-term outcome of patients with glycogen storage diseases," Journal of Inherited Metabolic Disease, vol. 13, no. 4, pp. 411-418, 1990.

[11] J. P. Rake, G. Visser, P. Labrune, J. V. Leonard, K. Ullrich, and G. P. A. Smit, "Glycogen storage disease type I: diagnosis, management, clinical course and outcome. Results of the European study on glycogen storage disease type I (ESGSD I)," European Journal of Pediatrics, vol. 161, no. 1, pp. S20-S34, 2002.

[12] H. R. Mundy, P. Georgiadou, L. C. Davies, A. Cousins, J. V. Leonard, and P. J. Lee, "Exercise capacity and biochemical profile during exercise in patients with glycogen storage disease type I," Journal of Clinical Endocrinology and Metabolism, vol. 90, no. 5, pp. 2675-2680, 2005.

[13] H. Özen, "Glycogen storage diseases: new perspectives," World Journal of Gastroenterology, vol. 13, no. 18, pp. 2541-2553, 2007.

[14] K. J. Lei, H. Chen, C. J. Pan et al., "Glucose-6-phosphatase dependent substrate transport in the glycogen storage disease type-1a mouse," Nature Genetics, vol. 13, no. 2, pp. 203-209, 1996.

[15] S. Y. Kim, L. Y. Chen, W. H. Yiu, D. A. Weinstein, and J. Y. Chou, "Neutrophilia and elevated serum cytokines are implicated in glycogen storage disease type Ia," FEBS Letters, vol. 581, no. 20, pp. 3833-3838, 2007.

[16] S. Y. Kim, D. A. Weinstein, M. F. Starost, B. C. Mansfield, and J. Y. Chou, "Necrotic foci, elevated chemokines and infiltrating neutrophils in the liver of glycogen storage disease type Ia," Journal of Hepatology, vol. 48, no. 3, pp. 479-485, 2008.

[17] A. E. Brix, E. W. Howerth, A. McConkie-Rosell et al., "Glycogen storage disease type Ia in two littermate Maltese puppies," Veterinary pathology, vol. 32, no. 5, pp. 460-465, 1995.

[18] P. S. Kishnani, Y. Bao, J. Y. Wu, A. E. Brix, JU. L. Lin, and Y. T. Chen, "Isolation and nucleotide sequence of canine glucose-6-phosphatase mRNA: identification of mutation in puppies with glycogen storage disease type Ia," Biochemical and Molecular Medicine, vol. 61, no. 2, pp. 168-177, 1997.
[19] P. S. Kishnani, E. Faulkner, S. VanCamp et al., "Canine model and genomic structural organization of glycogen storage disease type Ia (GSD Ia)," Veterinary Pathology, vol. 38, no. 1, pp. 83-91, 2001.

[20] K. J. Lei, C. J. Pan, L. L. Shelly, J. L. Liu, and J. Y. Chou, "Identification of mutations in the gene for glucose-6phosphatase, the enzyme deficient in glycogen storage disease type 1a," Journal of Clinical Investigation, vol. 93, no. 5, pp. 1994-1999, 1994.

[21] K. J. Lei, L. L. Shelly, C. J. Pan, J. B. Sidbury, and J. Y. Chou, "Mutations in the glucose-6-phosphatase gene that cause glycogen storage disease type 1a," Science, vol. 262, no. 5133, pp. 580-583, 1993.

[22] K. J. Lei, L. L. Shelly, B. Lin et al., "Mutations in the glucose-6phosphatase gene are associated with glycogen storage disease types $1 \mathrm{a}$ and $1 \mathrm{aSP}$ but not $1 \mathrm{~b}$ and 1c," Journal of Clinical Investigation, vol. 95, no. 1, pp. 234-240, 1995.

[23] K. J. Lei, Y. T. Chen, H. Chen et al., "Genetic basis of glycogen storage disease type 1a: prevalent mutations at the glucose-6phosphatase locus," American Journal of Human Genetics, vol. 57, no. 4, pp. 766-771, 1995.

[24] J. P. Rake, A. M. ten Berge, G. Visser et al., "Glycogen storage disease type Ia: recent experience with mutation analysis, a summary of mutations reported in the literature and a newly developed diagnostic flowchart," European Journal of Pediatrics, vol. 159, no. 5, pp. 322-330, 2000.

[25] R. N. Fine, S. D. Frasier, and G. N. Donnell, "Growth in glycogen-storage disease type 1. Evaluation of endocrine function," American Journal of Diseases of Children, vol. 117, no. 2, pp. 169-177, 1969.

[26] H. L. Greens, A. F. Slonim, J. A. O'Neill, and I. M. Burr, "Continuous nocturnal intragastric feeding for management of type I glycogen storage disease," The New England Journal of Medicine, vol. 294, no. 8, pp. 423-425, 1976.

[27] J. I. Wolfsdorf, R. A. Plotkin, L. M. B. Laffel, and J. F. Crigler, "Continuous glucose for treatment of patients with type 1 glycogen-storage disease: comparison of the effects of dextrose and uncooked cornstarch on biochemical variables," American Journal of Clinical Nutrition, vol. 52, no. 6, pp. 1043-1050, 1990.

[28] Y. T. Chen, M. Cornblath, and J. B. Sidbury, "Cornstarch therapy in type I glycogen-storage disease," The New England Journal of Medicine, vol. 310, no. 3, pp. 171-175, 1984.

[29] J. I. Wolfsdorf, C. R. Rudlin, and J. F. Crigler, "Physical growth and development of children with type 1 glycogenstorage disease: comparison of the effects of long-term use of dextrose and uncooked cornstarch," American Journal of Clinical Nutrition, vol. 52, no. 6, pp. 1051-1057, 1990.

[30] J. I. Wolfsdorf, S. Ehrlich, H. S. Landy, and J. F. Crigler, "Optimal daytime feeding regimen to prevent postprandial hypoglycemia in type 1 glycogen storage disease," American Journal of Clinical Nutrition, vol. 56, no. 3, pp. 587-592, 1992.

[31] C. E. Correia, K. Bhattacharya, P. J. Lee et al., "Use of modified cornstarch therapy to extend fasting in glycogen storage disease types Ia and Ib," American Journal of Clinical Nutrition, vol. 88, no. 5, pp. 1272-1276, 2008.

[32] R. M. Beaty, M. Jackson, D. Peterson et al., "Delivery of glucose-6-phosphatase in a canine model for glycogen storage disease, type la, with adeno-associated virus (AAV) vectors," Gene Therapy, vol. 9, no. 15, pp. 1015-1022, 2002.

[33] D. D. Koeberl, C. Pinto, B. Sun et al., "AAV vector-mediated reversal of hypoglycemia in canine and murine glycogen storage disease type Ia," Molecular Therapy, vol. 16, no. 4, pp. 665-672, 2008. 
[34] D. A. Weinstein, C. E. Correia, T. Conlon et al., "Adenoassociated virus-mediated correction of a canine model of glycogen storage disease type Ia," Human Gene Therapy, vol. 21, no. 7, pp. 903-910, 2010.

[35] J. Y. Chou and B. C. Mansfield, "Gene therapy for type I glycogen storage diseases," Current Gene Therapy, vol. 7, no. 2, pp. 79-88, 2007.

[36] W. H. Yiu, Y. M. Lee, W. T. Peng et al., "Complete normalization of hepatic G6PC deficiency in murine glycogen storage disease type Ia using gene therapy," Molecular Therapy, vol. 18, no. 6, pp. 1076-1084, 2010.

[37] D. D. Koeberl, P. S. Kishnani, and Y. T. Chen, "Glycogen storage disease types I and II: treatment updates," Journal of Inherited Metabolic Disease, vol. 30, no. 2, pp. 159-164, 2007.

[38] M. S. Sun, C. J. Pan, J. J. Shieh et al., "Sustained hepatic and renal glucose-6-phosphatase expression corrects glycogen storage disease type Ia in mice," Human Molecular Genetics, vol. 11, no. 18, pp. 2155-2164, 2002.

[39] Ad Hoc Committee on Dog and Cat Nutrition, Committee on Animal Nutrition, National Research Council, Nutrient Requirements of Dogs and Cats, National Academies Press, Washington, DC, USA, 2006.

[40] B. T. Kren, R. Metz, R. Kumar, and C. J. Steer, "Gene repair using chimeric RNA/DNA oligonucleotides," Seminars in Liver Disease, vol. 19, no. 1, pp. 93-104, 1999. 


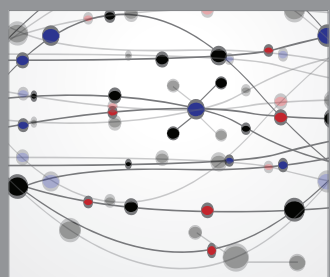

The Scientific World Journal
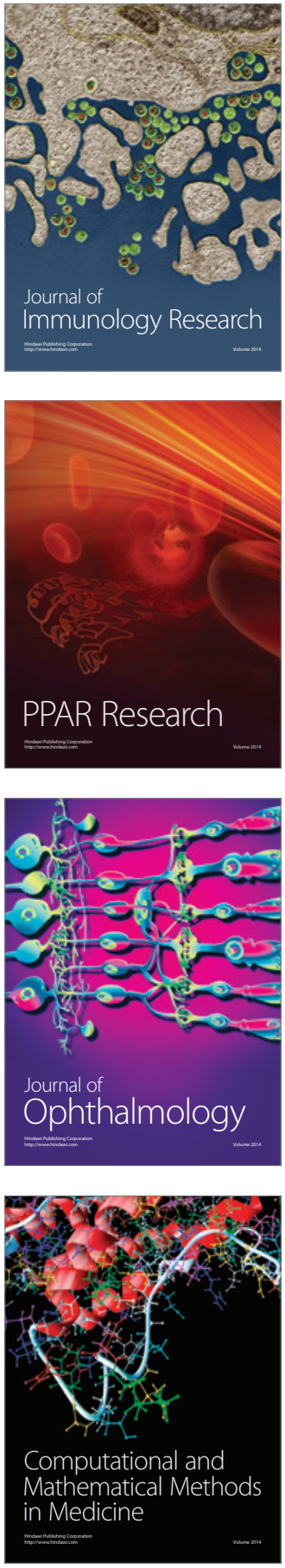

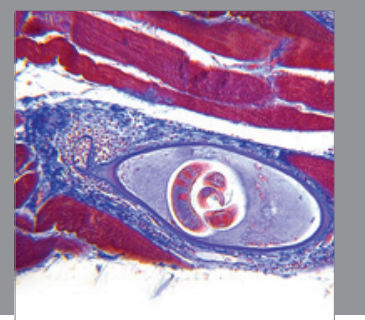

Gastroenterology

Research and Practice
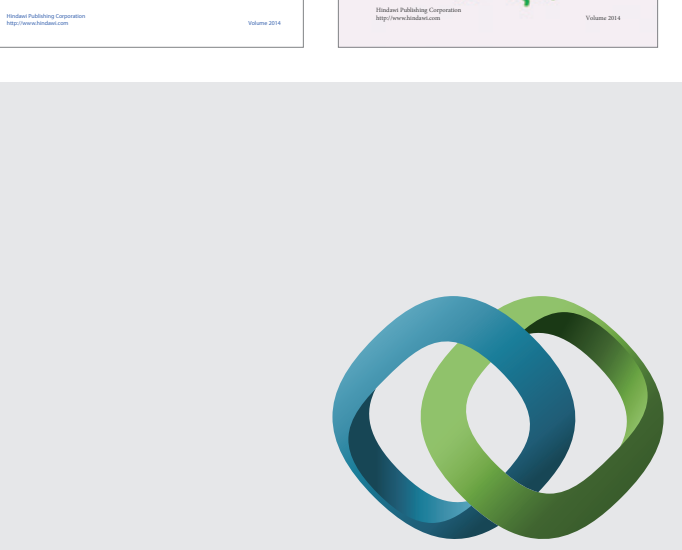

\section{Hindawi}

Submit your manuscripts at

http://www.hindawi.com
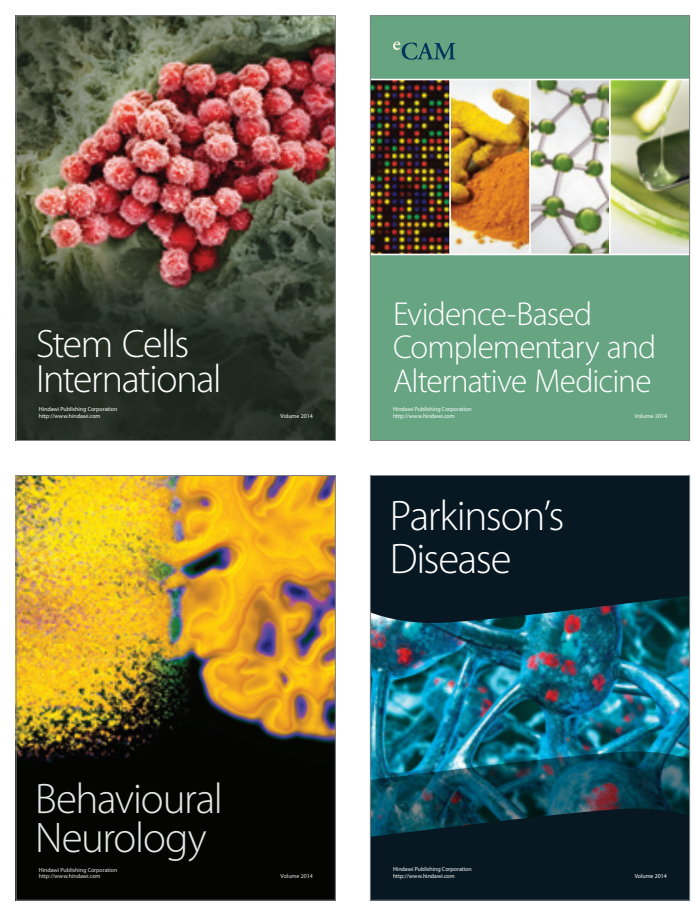

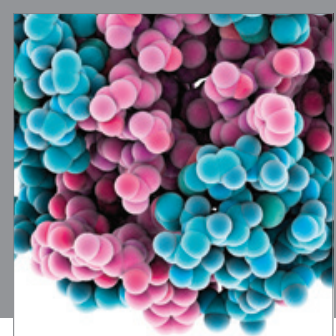

Journal of
Diabetes Research

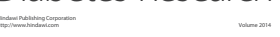

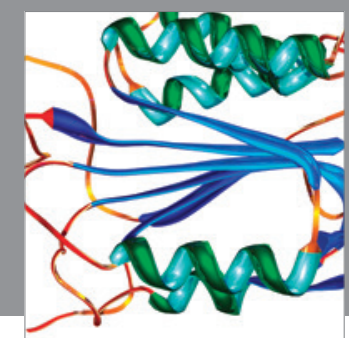

Disease Markers
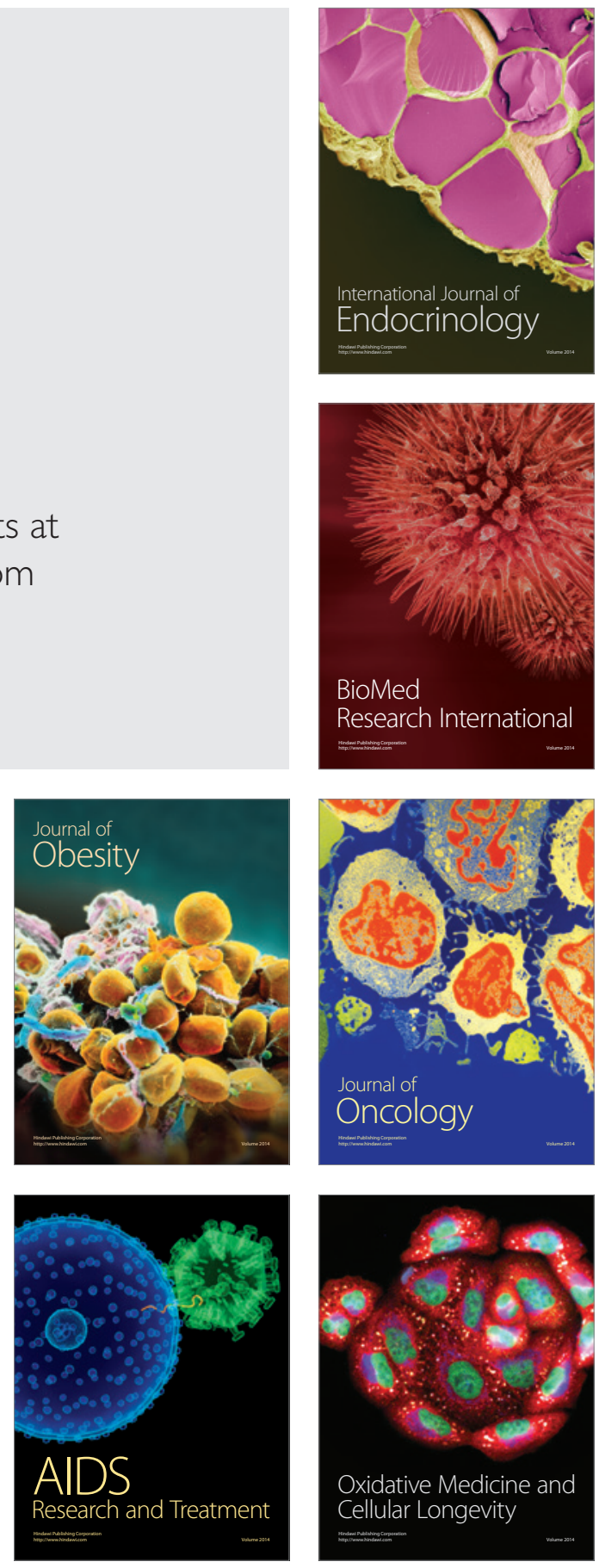